International Journal of Recent advances in Physics (IJRAP) Vol.4, No.4, November 2015

\title{
ON OPTIMIZATION OF MANUFACTURING OF MULTI- CHANNEL HETEROTRANSISTORS TO INCREASE THEIR INTEGRATION RATE
}

\author{
E.L. Pankratov ${ }^{1}$, E.A. Bulaeva ${ }^{1,2}$ \\ ${ }^{1}$ Nizhny Novgorod State University, 23 Gagarin avenue, Nizhny Novgorod, 603950, \\ Russia \\ ${ }^{2}$ Nizhny Novgorod State University of Architecture and Civil Engineering, 65 Il'insky \\ street, Nizhny Novgorod, 603950, Russia
}

\begin{abstract}
In this paper we consider an approach to increase integration rate of field-effect heterotransistors. Framework the approach we consider a heterostructure with specific configuration. After manufacturing the heterostructure we consider doping of required areas of the heterostructure by diffusion or ion implantation. The doping finished by optimized annealing of dopant and/or radiation defects. Framework this paper we consider a possibility to manufacture with several channels. Manufacturing multi-channel transistors gives us a possibility the to increase integration rate of transistors and to increase electrical current through the transistor.
\end{abstract}

\section{KEYWORDS}

Field-effect heterotransistors; multi-channel heterotransistors; increasing of integration rate

\section{INTRODUCTION}

In the present time integration rate of elements of integrated circuits intensively increasing [1-6]. Increasing of the integration rate leads to necessity to decrease dimensions of these elements. Are they widely using to decrease these elements laser and microwave types of annealing [7-11]. It could be also used radiation processing of doped materials to solve the same problem $[12,13]$.

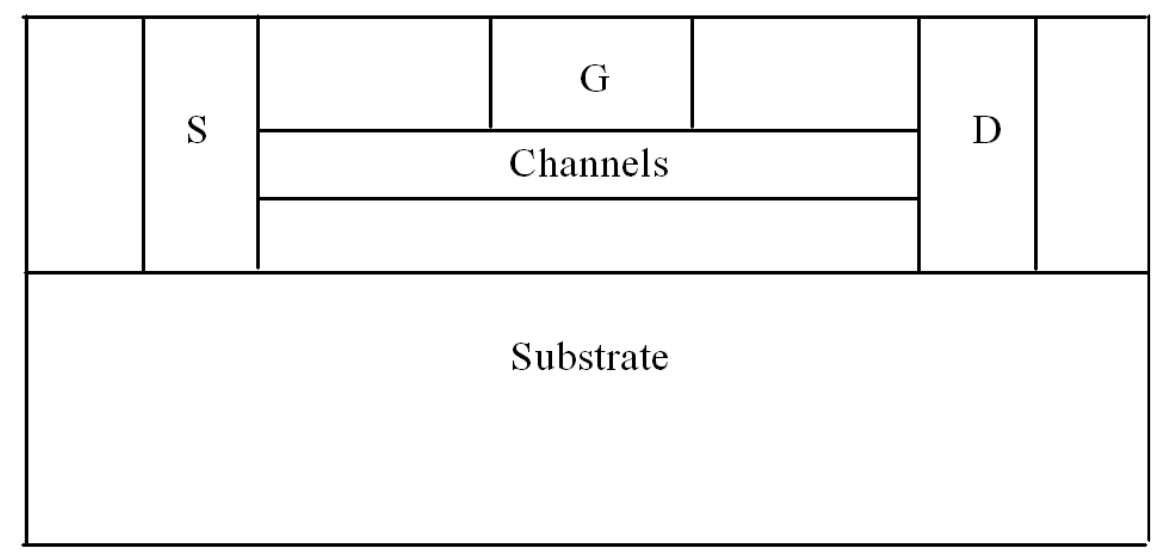

Fig. 1a. Heterostructure which includes into itself a substrate and an epitaxial layer. Side view DOI : 10.14810/ijrap.2015.4401 
In this paper we consider manufacturing a multi-channel heterotransistor with common gate. The heterotransistor has been manufactured framework a heterostructure, which include into itself a substrate and an epitaxial layer. The epitaxial layer includes into itself several sections (see Fig. 1). After manufacturing of the required quantity of channels they should be doped to generation required type of conductivity $(n$ or $p$ ). Farther we consider annealing of dopant and/or radiation defects. Increasing of annealing time leads to increasing of quantity of dopant in nearest materials. Decreasing of annealing time give not possibility to organize full doping of channels of transistors. Main aim of the present paper is analysis of spatio-temporal distributions of concentrations of dopant and radiation defects and temperature to determine conditions to decrease dimension of the considered heterotransistor.

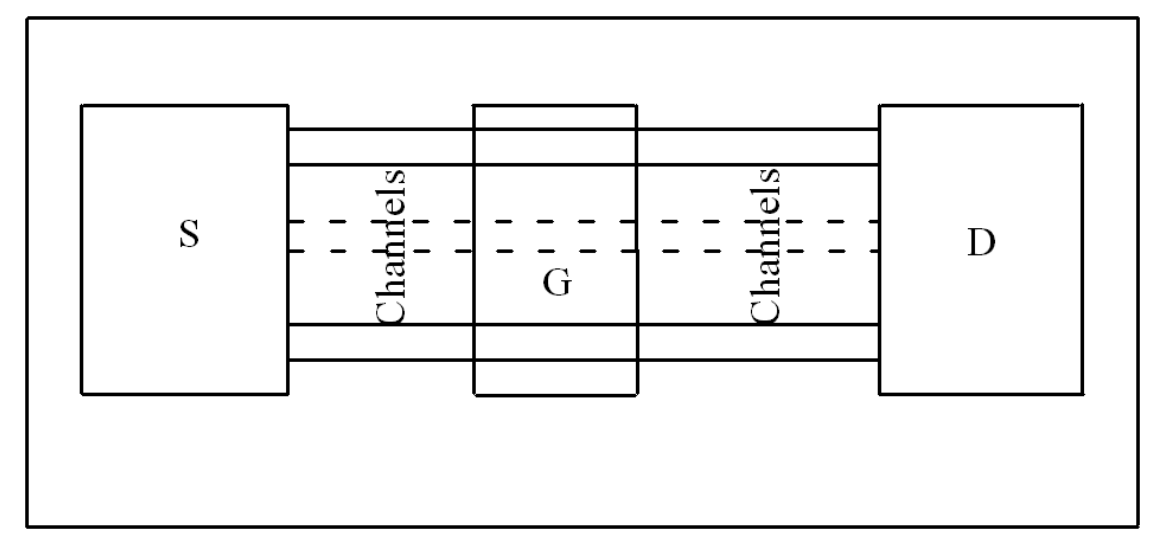

Fig. 1b. Heterostructure which includes into itself a substrate and an epitaxial layer. Top view

\section{METHOD OF SOLUTiON}

We solved our aim by calculation of spatio-temporal distributions of concentrations of dopant and radiation defects. We determine the required distribution of concentrations of dopant by solution of the following boundary problem

$$
\begin{gathered}
\frac{\partial C(x, y, z, t)}{\partial t}=\frac{\partial}{\partial x}\left[D_{C} \frac{\partial C(x, y, z, t)}{\partial x}\right]+\frac{\partial}{\partial y}\left[D_{C} \frac{\partial C(x, y, z, t)}{\partial y}\right]+\frac{\partial}{\partial z}\left[D_{C} \frac{\partial C(x, y, z, t)}{\partial z}\right] . \\
\left.\frac{\partial C(x, y, z, t)}{\partial x}\right|_{x=0}=0,\left.\frac{\partial C(x, y, z, t)}{\partial x}\right|_{x=L_{x}}=0,\left.\frac{\partial C(x, y, z, t)}{\partial y}\right|_{y=0}=0,\left.\frac{\partial C(x, y, z, t)}{\partial y}\right|_{x=L_{y}}=0 \\
\left.\frac{\partial C(x, y, z, t)}{\partial z}\right|_{z=0}=0,\left.\frac{\partial C(x, y, z, t)}{\partial z}\right|_{x=L_{z}}=0, C(x, y, z, 0)=f(x, y, z) .
\end{gathered}
$$

Function $C(x, y, z, t)$ describes the spatio-temporal distribution of concentration of dopant; $T$ is the temperature of annealing; $D_{C}$ is the dopant diffusion coefficient. Dopant diffusion coefficient will have different values in different materials. The value will be changed during heating and cooling of heterostructure (with account Arrhenius law). Approximation of dopant diffusion coefficient by the following function [13-15]

$$
D_{C}=D_{L}(x, y, z, T)\left[1+\xi \frac{C^{\gamma}(x, y, z, t)}{P^{\gamma}(x, y, z, T)}\right]\left[1+\varsigma_{1} \frac{V(x, y, z, t)}{V^{*}}+\varsigma_{2} \frac{V^{2}(x, y, z, t)}{\left(V^{*}\right)^{2}}\right] .
$$


Function $D_{L}(x, y, z, T)$ describes the spatial and temperature dependences of dopant diffusion coefficient. One can find these dependences due to presents several layers in heterostructure and the Arrhenius law, respectively. Function $P(x, y, z, T)$ describes the limit of solubility of dopant. Parameter $\gamma \in[1,3]$ has different values in different materials [14]. The function $V(x, y, z, t)$ describes the spatio-temporal distribution of concentration of radiation vacancies. $V^{*}$ is the equilibrium distribution of concentration of vacancies. Dependence of dopant diffusion coefficient on concentration of dopant has been described in details in [14]. It is known, that doping of materials by diffusion did not leads to generation radiation defects. In this situation $\zeta_{1}=\zeta_{2}=0$. It should be also noted, that we solved more common boundary problem in comparison with recently published works [1-15].

We determine spatio-temporal distributions of concentrations of radiation defects by solving the following boundary problem $[13,15]$

$$
\begin{aligned}
& \frac{\partial I(x, y, z, t)}{\partial t}=\frac{\partial}{\partial x}\left[D_{I}(x, y, z, T) \frac{\partial I(x, y, z, t)}{\partial x}\right]+\frac{\partial}{\partial y}\left[D_{I}(x, y, z, T) \frac{\partial I(x, y, z, t)}{\partial y}\right]-k_{I, I}(x, y, z, T) \times \\
& \quad \times I^{2}(x, y, z, t)+\frac{\partial}{\partial z}\left[D_{I}(x, y, z, T) \frac{\partial I(x, y, z, t)}{\partial z}\right]-k_{I, V}(x, y, z, T) I(x, y, z, t) V(x, y, z, t) \\
& \frac{\partial V(x, y, z, t)}{\partial t}=\frac{\partial}{\partial x}\left[D_{V}(x, y, z, T) \frac{\partial V(x, y, z, t)}{\partial x}\right]+\frac{\partial}{\partial y}\left[D_{V}(x, y, z, T) \frac{\partial V(x, y, z, t)}{\partial y}\right]-k_{V, V}(x, y, z, T) \times \\
& \quad \times V^{2}(x, y, z, t)+\frac{\partial}{\partial z}\left[D_{V}(x, y, z, T) \frac{\partial V(x, y, z, t)}{\partial z}\right]-k_{I, V}(x, y, z, T) I(x, y, z, t) V(x, y, z, t) .
\end{aligned}
$$

Boundary and initial conditions for these equations are

$$
\begin{gathered}
\left.\frac{\partial \rho(x, y, z, t)}{\partial x}\right|_{x=0}=0,\left.\frac{\partial \rho(x, y, z, t)}{\partial x}\right|_{x=L_{x}}=0,\left.\frac{\partial \rho(x, y, z, t)}{\partial y}\right|_{y=0}=0,\left.\frac{\partial \rho(x, y, z, t)}{\partial y}\right|_{y=L_{y}}=0, \\
\left.\frac{\partial \rho(x, y, z, t)}{\partial z}\right|_{z=0}=0,\left.\frac{\partial \rho(x, y, z, t)}{\partial z}\right|_{z=L_{z}}=0, \rho(x, y, z, 0)=f_{\rho}(x, y, z) .
\end{gathered}
$$

Here $\rho=I, V$. The function $I(x, y, z, t)$ describes the spatio-temporal distribution of concentration of radiation interstitials. The functions $D_{\rho}(x, y, z, T)$ describes the approximations of diffusion coefficients of point radiation defects. Terms $V^{2}(x, y, z, t)$ and $I^{2}(x, y, z, t)$ correspond to generation divacancies and diinterstitials. The function $k_{I, V}(x, y, z, T)$ describes the parameter of recombination of point radiation defects. The functions $k_{I, I}(x, y, z, T)$ and $k_{V, V}(x, y, z, T)$ describe the parameters of generation of simplest complexes of point radiation defects.

Now we calculate distributions of concentrations of divacancies $\Phi_{V}(x, y, z, t)$ and diinterstitials $\Phi_{I}(x, y, z, t)$ in space and time by solving the following boundary problem $[13,15]$

$$
\begin{aligned}
& \frac{\partial \Phi_{I}(x, y, z, t)}{\partial t}=\frac{\partial}{\partial x}\left[D_{\Phi I}(x, y, z, T) \frac{\partial \Phi_{I}(x, y, z, t)}{\partial x}\right]+\frac{\partial}{\partial y}\left[D_{\Phi I}(x, y, z, T) \frac{\partial \Phi_{I}(x, y, z, t)}{\partial y}\right]+ \\
& +\frac{\partial}{\partial z}\left[D_{\Phi I}(x, y, z, T) \frac{\partial \Phi_{I}(x, y, z, t)}{\partial z}\right]+k_{I, I}(x, y, z, T) I^{2}(x, y, z, t)-k_{I}(x, y, z, T) I(x, y, z, t)
\end{aligned}
$$


International Journal of Recent advances in Physics (IJRAP) Vol.4, No.4, November 2015

$$
\begin{gathered}
\frac{\partial \Phi_{V}(x, y, z, t)}{\partial t}=\frac{\partial}{\partial x}\left[D_{\Phi V}(x, y, z, T) \frac{\partial \Phi_{V}(x, y, z, t)}{\partial x}\right]+\frac{\partial}{\partial y}\left[D_{\Phi V}(x, y, z, T) \frac{\partial \Phi_{V}(x, y, z, t)}{\partial y}\right]+ \\
+\frac{\partial}{\partial z}\left[D_{\Phi V}(x, y, z, T) \frac{\partial \Phi_{V}(x, y, z, t)}{\partial z}\right]+k_{V, V}(x, y, z, T) V^{2}(x, y, z, t)-k_{V}(x, y, z, T) V(x, y, z, t), \\
\left.\frac{\partial \Phi_{\rho}(x, y, z, t)}{\partial x}\right|_{x=0}=0,\left.\frac{\partial \Phi_{\rho}(x, y, z, t)}{\partial x}\right|_{x=L_{x}}=0,\left.\frac{\partial \Phi_{\rho}(x, y, z, t)}{\partial y}\right|_{y=0}=0,\left.\frac{\partial \Phi_{\rho}(x, y, z, t)}{\partial y}\right|_{y=L_{y}}=0, \\
\left.\frac{\partial \Phi_{\rho}(x, y, z, t)}{\partial z}\right|_{z=0}=0,\left.\frac{\partial \Phi_{\rho}(x, y, z, t)}{\partial z}\right|_{z=L_{z}}=0, \Phi_{I}(x, y, z, 0)=f_{\Phi I}(x, y, z), \Phi_{V}(x, y, z, 0)=f_{\Phi V}(x, y, z) .(7)
\end{gathered}
$$

The functions $D_{\Phi \rho}(x, y, z, T)$ describe the diffusion coefficients of the above complexes of radiation defects. The functions $k_{I}(x, y, z, T)$ and $k_{V}(x, y, z, T)$ describe the parameters of decay of these complexes. In this situation boundary problems Eqs.(4)-(7) are generalization of analogous problems in [13-15].

We determine distribution of temperature in space and time as solution of the second law of Fourier [14]

$$
\begin{aligned}
c(T) \frac{\partial T(x, y, z, t)}{\partial t}= & \frac{\partial}{\partial x}\left[\lambda(x, y, z, T) \frac{\partial T(x, y, z, t)}{\partial x}\right]+\frac{\partial}{\partial y}\left[\lambda(x, y, z, T) \frac{\partial T(x, y, z, t)}{\partial y}\right]+ \\
& +\frac{\partial}{\partial z}\left[\lambda(x, y, z, T) \frac{\partial T(x, y, z, t)}{\partial z}\right]+p(x, y, z, t) .
\end{aligned}
$$

Boundary and initial conditions for the Eq. (8) are

$$
\begin{gathered}
\left.\frac{\partial T(x, y, z, t)}{\partial x}\right|_{x=0}=0,\left.\frac{\partial T(x, y, z, t)}{\partial x}\right|_{x=L_{x}}=0,\left.\frac{\partial T(x, y, z, t)}{\partial y}\right|_{y=0}=0,\left.\frac{\partial T(x, y, z, t)}{\partial y}\right|_{x=L_{y}}=0, \\
\left.\frac{\partial T(x, y, z, t)}{\partial z}\right|_{z=0}=0,\left.\frac{\partial T(x, y, z, t)}{\partial z}\right|_{x=L_{z}}=0, T(x, y, z, 0)=f_{T}(x, y, z),
\end{gathered}
$$

Function $T(x, y, z, t)$ describes distribution of temperature in space and time during annealing of dopant and/or radiation defects. Function $c(T)=c_{a s s}\left[1-\eta \exp \left(-T(x, y, z, t) / T_{d}\right)\right]$ describes dependence of the heat capacitance on temperature. In the considered situation current temperature is approximately equal or larger, than Debye temperature $T_{d}$. In this situation we have a possibility to consider the following limiting case $c(T) \approx c_{\text {ass }}$ ) [14]. The function $\lambda$ describes the heat conduction coefficient. The heat conduction coefficient depends on properties of materials and temperature and could be approximated by the following function $\lambda(x, y, z, T)=\lambda_{a s s}(x, y, z)\left[1+\mu\left(T_{d} / T(x, y, z, t)\right)^{\varphi}\right]$ [14]). The function describes $p(x, y, z, t)$ is the volumetric density of power of heating; $\alpha$ $(x, y, z, T)=\lambda(x, y, z, T) / c(T)$ is the thermal diffusivity.

We calculate distribution of concentrations of dopant and radiation defects and temperature in space and time by method of averaging of function corrections [16-18] with decreasing quantity of iteration steps [17]. To use the approach we consider solutions of Eqs. (1), (4), (6), (8) with averaged values of diffusion coefficients $D_{0 L}, D_{0 I}, D_{0 V}, D_{0 \Phi I}, D_{0 \Phi V}$, zero values of parameters of recombination of radiation defects and parameters of generation and decay of their complexes as initial-order approximations of required concentrations. The initial-order approximations could be written as 


$$
\begin{aligned}
& C_{1}(x, y, z, t)=\frac{1}{L_{x} L_{y} L_{z}}+\frac{2}{L_{x} L_{y} L_{z}} \sum_{n=1}^{\infty} F_{n C} c_{n}(x) c_{n}(y) c_{n}(z) e_{n C}(t), \\
& I_{1}(x, y, z, t)=\frac{1}{L_{x} L_{y} L_{z}}+\frac{2}{L_{x} L_{y} L_{z}} \sum_{n=1}^{\infty} F_{n I} c_{n}(x) c_{n}(y) c_{n}(z) e_{n I}(t), \\
& V_{1}(x, y, z, t)=\frac{1}{L_{x} L_{y} L_{z}}+\frac{2}{L_{x} L_{y} L_{z}} \sum_{n=1}^{\infty} F_{n V} c_{n}(x) c_{n}(y) c_{n}(z) e_{n V}(t), \\
& \Phi_{I 1}(x, y, z, t)=\frac{1}{L_{x} L_{y} L_{z}}+\frac{2}{L_{x} L_{y} L_{z}} \sum_{n=1}^{\infty} F_{n \Phi_{I}} c_{n}(x) c_{n}(y) c_{n}(z) e_{n \Phi_{I}}(t), \\
& \Phi_{V 1}(x, y, z, t)=\frac{1}{L_{x} L_{y} L_{z}}+\frac{2}{L_{x} L_{y} L_{z}} \sum_{n=1}^{\infty} F_{n \Phi_{V}} c_{n}(x) c_{n}(y) c_{n}(z) e_{n \Phi_{V}}(t), \\
& T_{1}(x, y, z, t)=\frac{1}{L_{x} L_{y} L_{z}}+\frac{2}{L_{x} L_{y} L_{z}} \sum_{n=1}^{\infty} F_{n T} c_{n}(x) c_{n}(y) c_{n}(z) e_{n T}(t), \\
& \text { where } e_{n \rho}(t)=\exp \left[-\pi^{2} n^{2} D_{0 \rho} t\left(\frac{1}{L_{x}^{2}}+\frac{1}{L_{y}^{2}}+\frac{1}{L_{z}^{2}}\right)\right], F_{n \rho}=\int_{0}^{L_{x}} c_{n}(u) \int_{0}^{L_{y}} c_{n}(v) \int_{0}^{L_{z}} c_{n}(w) f_{\rho}(u, v, w) d w d v d u \text {, } \\
& e_{n T}(t)=\exp \left[-\pi^{2} n^{2} \alpha_{0} t\left(\frac{1}{L_{x}^{2}}+\frac{1}{L_{y}^{2}}+\frac{1}{L_{z}^{2}}\right)\right], c_{n}(\chi)=\cos \left(\pi n \chi / L_{\chi}\right) .
\end{aligned}
$$

One could calculate approximations of the second- and other orders framework classical iteration procedure of method of averaging of function corrections [16-18]. Framework this procedure to determine the $n$-th-order approximation of concentrations of dopant and radiation defects we shall replace the required functions $C(x, y, z, t), I(x, y, z, t), V(x, y, z, t), \Phi_{I}(x, y, z, t), \Phi_{V}(x, y, z, t)$ and $T(x, y, z, t)$ in the right sides of Eqs. (1), (4), (6), (8) on the following sums $\alpha_{n \rho}+\rho_{n-1}(x, y, z, t)$, when $\alpha_{n \rho}$ are not yet known average values of the $n$-th-order approximation of the above concentrations. The replacement leads to the following relations for the second-order approximations of the required concentrations

$$
\begin{gathered}
\frac{\partial C_{2}(x, y, z, t)}{\partial t}=\frac{\partial}{\partial x}\left(D_{L}(x, y, z, T)\left[1+\varsigma_{1} \frac{V(x, y, z, t)}{V^{*}}+\varsigma_{2} \frac{V^{2}(x, y, z, t)}{\left(V^{*}\right)^{2}}\right] \frac{\partial C_{1}(x, y, z, t)}{\partial x} \times\right. \\
\left.\times\left\{1+\xi \frac{\left[\alpha_{2 C}+C_{1}(x, y, z, t)\right]^{\gamma}}{P^{\gamma}(x, y, z, T)}\right\}\right)+\frac{\partial}{\partial y}\left(D_{L}(x, y, z, T)\left[1+\varsigma_{1} \frac{V(x, y, z, t)}{V^{*}}+\varsigma_{2} \frac{V^{2}(x, y, z, t)}{\left(V^{*}\right)^{2}}\right] \times\right. \\
\left.\times\left\{1+\xi \frac{\left[\alpha_{2 C}+C_{1}(x, y, z, t)\right]^{\gamma}}{P^{\gamma}(x, y, z, T)}\right\} \frac{\partial C_{1}(x, y, z, t)}{\partial y}\right)+\frac{\partial}{\partial z}\left(\left\{1+\xi \frac{\left[\alpha_{2 C}+C_{1}(x, y, z, t)\right]^{\gamma}}{P^{\gamma}(x, y, z, T)}\right\} \times\right. \\
\left.\times D_{L}(x, y, z, T)\left[1+\varsigma_{1} \frac{V(x, y, z, t)}{V^{*}}+\varsigma_{2} \frac{V^{2}(x, y, z, t)}{\left(V^{*}\right)^{2}}\right] \frac{\partial C_{1}(x, y, z, t)}{\partial z}\right)
\end{gathered}
$$


International Journal of Recent advances in Physics (IJRAP) Vol.4, No.4, November 2015

$$
\begin{aligned}
& \frac{\partial I_{2}(x, y, z, t)}{\partial t}=\frac{\partial}{\partial x}\left[D_{I}(x, y, z, T) \frac{\partial I_{1}(x, y, z, t)}{\partial x}\right]+\frac{\partial}{\partial y}\left[D_{I}(x, y, z, T) \frac{\partial I_{1}(x, y, z, t)}{\partial y}\right]+ \\
& +\frac{\partial}{\partial z}\left[D_{I}(x, y, z, T) \frac{\partial I_{1}(x, y, z, t)}{\partial z}\right]-k_{I, V}(x, y, z, T)\left[\alpha_{2 I}+I_{1}(x, y, z, t)\right]\left[\alpha_{2 V}+V_{1}(x, y, z, t)\right]- \\
& -k_{I, I}(x, y, z, T)\left[\alpha_{2 I}+I_{1}(x, y, z, t)\right]^{2} \\
& \frac{\partial V_{2}(x, y, z, t)}{\partial t}=\frac{\partial}{\partial x}\left[D_{V}(x, y, z, T) \frac{\partial V_{1}(x, y, z, t)}{\partial x}\right]+\frac{\partial}{\partial y}\left[D_{V}(x, y, z, T) \frac{\partial V_{1}(x, y, z, t)}{\partial y}\right]+ \\
& +\frac{\partial}{\partial z}\left[D_{V}(x, y, z, T) \frac{\partial V_{1}(x, y, z, t)}{\partial z}\right]-k_{I, V}(x, y, z, T)\left[\alpha_{2 I}+I_{1}(x, y, z, t)\right]\left[\alpha_{2 V}+V_{1}(x, y, z, t)\right]- \\
& -k_{V, V}(x, y, z, T)\left[\alpha_{2 V}+V_{1}(x, y, z, t)\right]^{2} \\
& \frac{\partial \Phi_{I 2}(x, y, z, t)}{\partial t}=\frac{\partial}{\partial x}\left[D_{\Phi I}(x, y, z, T) \frac{\partial \Phi_{I 1}(x, y, z, t)}{\partial x}\right]+k_{I, I}(x, y, z, T) I^{2}(x, y, z, t)+ \\
& +\frac{\partial}{\partial y}\left[D_{\Phi I}(x, y, z, T) \frac{\partial \Phi_{I 1}(x, y, z, t)}{\partial y}\right]+\frac{\partial}{\partial z}\left[D_{\Phi I}(x, y, z, T) \frac{\partial \Phi_{I 1}(x, y, z, t)}{\partial z}\right]- \\
& -k_{I}(x, y, z, T) I(x, y, z, t) \\
& \frac{\partial \Phi_{V 2}(x, y, z, t)}{\partial t}=\frac{\partial}{\partial x}\left[D_{\Phi V}(x, y, z, T) \frac{\partial \Phi_{V 1}(x, y, z, t)}{\partial x}\right]+k_{V, V}(x, y, z, T) V^{2}(x, y, z, t)+ \\
& +\frac{\partial}{\partial y}\left[D_{\Phi V}(x, y, z, T) \frac{\partial \Phi_{V 1}(x, y, z, t)}{\partial y}\right]+\frac{\partial}{\partial z}\left[D_{\Phi V}(x, y, z, T) \frac{\partial \Phi_{V 1}(x, y, z, t)}{\partial z}\right]- \\
& -k_{V}(x, y, z, T) V(x, y, z, t) \text {. } \\
& c \frac{\partial T_{2}(x, y, z, t)}{\partial t}=\frac{\partial}{\partial x}\left(\lambda_{a s s}(x, y, z)\left\{1+\mu\left[\frac{T_{d}}{T(x, y, z, t)}\right]^{\varphi}\right\} \frac{\partial\left[\alpha_{2 T}+T_{1}(x, y, z, t)\right]}{\partial x}\right)+ \\
& +\frac{\partial}{\partial y}\left(\lambda_{\text {ass }}(x, y, z)\left\{1+\mu\left[\frac{T_{d}}{T(x, y, z, t)}\right]^{\varphi}\right\} \frac{\partial\left[\alpha_{2 T}+T_{1}(x, y, z, t)\right]}{\partial y}\right)+ \\
& +\frac{\partial}{\partial z}\left(\lambda_{\text {ass }}(x, y, z)\left\{1+\mu\left[\frac{T_{d}}{T(x, y, z, t)}\right]^{\varphi}\right\} \frac{\partial\left[\alpha_{2 T}+T_{1}(x, y, z, t)\right]}{\partial z}\right)+p(x, y, z, t) .
\end{aligned}
$$

Integration of both sides of Eqs. (10)-(13) on time gives us possibility to obtain relations for the second-order approximations of the required concentrations in the final form 
International Journal of Recent advances in Physics (IJRAP) Vol.4, No.4, November 2015

$$
\begin{aligned}
& C_{2}(x, y, z, t)=\frac{\partial}{\partial x}\left(\int_{0}^{t} D_{L}(x, y, z, T)\left[1+\varsigma_{1} \frac{V(x, y, z, \tau)}{V^{*}}+\varsigma_{2} \frac{V^{2}(x, y, z, \tau)}{\left(V^{*}\right)^{2}}\right] \frac{\partial C_{1}(x, y, z, \tau)}{\partial x} \times\right. \\
& \left.\times\left\{1+\xi \frac{\left[\alpha_{2 C}+C_{1}(x, y, z, \tau)\right]^{\gamma}}{P^{\gamma}(x, y, z, T)}\right\}\right) d \tau+\frac{\partial}{\partial y}\left(\int_{0}^{t}\left[1+\varsigma_{1} \frac{V(x, y, z, \tau)}{V^{*}}+\varsigma_{2} \frac{V^{2}(x, y, z, \tau)}{\left(V^{*}\right)^{2}}\right] \times\right. \\
& \left.\times D_{L}(x, y, z, T)\left\{1+\xi \frac{\left[\alpha_{2 C}+C_{1}(x, y, z, \tau)\right]^{\gamma}}{P^{\gamma}(x, y, z, T)}\right\} \frac{\partial C_{1}(x, y, z, \tau)}{\partial y} d \tau\right)+\frac{\partial}{\partial z}\left(\int_{0}^{t} \frac{\partial C_{1}(x, y, z, \tau)}{\partial z} \times\right. \\
& \left.\times D_{L}(x, y, z, T)\left[1+\varsigma_{1} \frac{V(x, y, z, \tau)}{V^{*}}+\varsigma_{2} \frac{V^{2}(x, y, z, \tau)}{\left(V^{*}\right)^{2}}\right] d \tau\right)+f_{C}(x, y, z) \\
& I_{2}(x, y, z, t)=\frac{\partial}{\partial x}\left[\int_{0}^{t} D_{I}(x, y, z, T) \frac{\partial I_{1}(x, y, z, \tau)}{\partial x} d \tau\right]+\frac{\partial}{\partial y}\left[\int_{0}^{t} D_{I}(x, y, z, T) \frac{\partial I_{1}(x, y, z, \tau)}{\partial y} d \tau\right]+ \\
& +\frac{\partial}{\partial z}\left[\int_{0}^{t} D_{I}(x, y, z, T) \frac{\partial I_{1}(x, y, z, \tau)}{\partial z} d \tau\right]-\int_{0}^{t} k_{I, I}(x, y, z, T)\left[\alpha_{2 I}+I_{1}(x, y, z, \tau)\right]^{2} d \tau- \\
& -\int_{0}^{t} k_{I, V}(x, y, z, T)\left[\alpha_{2 I}+I_{1}(x, y, z, \tau)\right]\left[\alpha_{2 V}+V_{1}(x, y, z, \tau)\right] d \tau+f_{I}(x, y, z) \\
& V_{2}(x, y, z, t)=\frac{\partial}{\partial x}\left[\int_{0}^{t} D_{V}(x, y, z, T) \frac{\partial V_{1}(x, y, z, \tau)}{\partial x} d \tau\right]+\frac{\partial}{\partial y}\left[\int_{0}^{t} D_{V}(x, y, z, T) \frac{\partial V_{1}(x, y, z, \tau)}{\partial y} d \tau\right]+ \\
& +\frac{\partial}{\partial z}\left[\int_{0}^{t} D_{V}(x, y, z, T) \frac{\partial V_{1}(x, y, z, \tau)}{\partial z} d \tau\right]-\int_{0}^{t} k_{V, V}(x, y, z, T)\left[\alpha_{2 I}+V_{1}(x, y, z, \tau)\right]^{2} d \tau- \\
& -\int_{0}^{t} k_{I, V}(x, y, z, T)\left[\alpha_{2 I}+I_{1}(x, y, z, \tau)\right]\left[\alpha_{2 V}+V_{1}(x, y, z, \tau)\right] d \tau+f_{V}(x, y, z) \\
& \Phi_{I 2}(x, y, z, t)=\frac{\partial}{\partial x}\left[\int_{0}^{t} D_{\Phi I}(x, y, z, T) \frac{\partial \Phi_{I 1}(x, y, z, \tau)}{\partial x} d \tau\right]-\int_{0}^{t} k_{I}(x, y, z, T) I(x, y, z, \tau) d \tau+ \\
& +\frac{\partial}{\partial y}\left[\int_{0}^{t} D_{\Phi I}(x, y, z, T) \frac{\partial \Phi_{I 1}(x, y, z, \tau)}{\partial y} d \tau\right]+\frac{\partial}{\partial z}\left[\int_{0}^{t} D_{\Phi I}(x, y, z, T) \frac{\partial \Phi_{I 1}(x, y, z, \tau)}{\partial z} d \tau\right]+ \\
& +\int_{0}^{t} k_{I, I}(x, y, z, T) I^{2}(x, y, z, \tau) d \tau+f_{\Phi I}(x, y, z) \\
& \Phi_{V 2}(x, y, z, t)=\frac{\partial}{\partial x}\left[\int_{0}^{t} D_{\Phi V}(x, y, z, T) \frac{\partial \Phi_{V 1}(x, y, z, \tau)}{\partial x} d \tau\right]-\int_{0}^{t} k_{V}(x, y, z, T) V(x, y, z, \tau) d \tau+
\end{aligned}
$$


International Journal of Recent advances in Physics (IJRAP) Vol.4, No.4, November 2015

$$
\begin{aligned}
& +\frac{\partial}{\partial y}\left[\int_{0}^{t} D_{\Phi V}(x, y, z, T) \frac{\partial \Phi_{V 1}(x, y, z, \tau)}{\partial y} d \tau\right]+\frac{\partial}{\partial z}\left[\int_{0}^{t} D_{\Phi V}(x, y, z, T) \frac{\partial \Phi_{V 1}(x, y, z, \tau)}{\partial z} d \tau\right]+ \\
& +\int_{0}^{t} k_{V, V}(x, y, z, T) V^{2}(x, y, z, \tau) d \tau+f_{\Phi V}(x, y, z), \\
& c T(x, y, z, t)=\frac{\partial}{\partial x}\left(\lambda_{\text {ass }}(x, y, z) \int_{0}^{t}\left\{1+\mu\left[\frac{T_{d}}{T(x, y, z, \tau)}\right]^{\varphi}\right\} \frac{\partial\left[\alpha_{2 T}+T_{1}(x, y, z, \tau)\right]}{\partial x} d \tau\right)+ \\
& +f_{T}(x, y, z)+\frac{\partial}{\partial y}\left(\lambda_{a s s}(x, y, z) \int_{0}^{t}\left\{1+\mu\left[\frac{T_{d}}{T(x, y, z, \tau)}\right]^{\varphi}\right\} \frac{\partial\left[\alpha_{2 T}+T_{1}(x, y, z, \tau)\right]}{\partial y} d \tau\right)+ \\
& +\frac{\partial}{\partial z}\left(\lambda_{a s s}(x, y, z) \int_{0}^{t}\left\{1+\mu\left[\frac{T_{d}}{T(x, y, z, \tau)}\right]\right]^{\varphi}\left\{\frac{\partial\left[\alpha_{2 T}+T_{1}(x, y, z, \tau)\right]}{\partial z} d \tau\right)+\int_{0}^{t} p(x, y, z, \tau) d \tau .\right.
\end{aligned}
$$

One could calculate average values of the second-orders approximations of the required concentrations and temperature by the standard relation [16-18]

$$
\alpha_{2 \rho}=\frac{1}{\Theta L_{x} L_{y} L_{z}} \int_{0}^{\Theta} \int_{0}^{L_{x}} \int_{0}^{L_{y} L_{z}} \int_{0}\left[\rho_{2}(x, y, z, t)-\rho_{1}(x, y, z, t)\right] d z d y d x d t .
$$

Final relations for the required average values by substitution of the relations $(10 a)-(13 a)$ into the relation (14)

$$
\begin{aligned}
& \alpha_{2 C}=\frac{1}{L_{x} L_{y} L_{z}} \int_{0}^{L_{x} L_{y} L_{z}} \int_{0}^{0} f_{C}(x, y, z) d z d y d x \\
& \alpha_{2 I}=\frac{1}{2 A_{I I 00}}\left\{\left(1+A_{I V 01}+A_{I I 10}+\alpha_{2 V} A_{I V 00}\right)^{2}-4 A_{I I 00}\left[\alpha_{2 V} A_{I V 10}-A_{I I 20}+A_{I V 11}-\right.\right. \\
& \left.\left.-\frac{1}{L_{x} L_{y} L_{z}} \int_{0}^{L_{x}} \int_{0}^{L_{y} L_{z}} \int_{0} f_{I}(x, y, z) d z d y d x\right]\right\}^{\frac{1}{2}}-\frac{1+A_{I V 01}+A_{I I 10}+\alpha_{2 V} A_{I V 00}}{2 A_{I I 00}}, \\
& \alpha_{2 V}=\frac{1}{2 B_{4}} \sqrt{\frac{\left(B_{3}+A\right)^{2}}{4}-4 B_{4}\left(y+\frac{B_{3} y-B_{1}}{A}\right)}-\frac{B_{3}+A}{4 B_{4}} .
\end{aligned}
$$

Parameters $A_{a b i j}$ and other parameters in the relations (16) are presented in the Appendix. Parameters $\alpha_{a b i j}$ and other parameters in the relations (16) could be written as

$$
\begin{aligned}
\alpha_{2 \Phi_{I}}=A_{I I 20}-\frac{1}{\Theta L_{x} L_{y} L_{z}} \int_{0}^{\Theta}(\Theta-t) \int_{0}^{L_{x}} \int_{0}^{L_{y} L_{z}} \int_{0} k_{I}(x, y, z, T) I(x, y, z, t) d z d y d x d t+ \\
\quad+\frac{1}{L_{x} L_{y} L_{z}} \int_{0}^{L_{x} L_{y} L_{z}} \int_{0} \int_{0} f_{\Phi I}(x, y, z) d z d y d x
\end{aligned}
$$




$$
\begin{aligned}
\alpha_{2 \Phi_{V}}=A_{V V 20}-\frac{1}{\Theta L_{x} L_{y} L_{z}} \int_{0}^{\Theta}(\Theta-t) \int_{0}^{L_{x}} \int_{0}^{L_{y} L_{z}} \int_{0} k_{V}(x, y, z, T) V(x, y, z, t) d z d y d x d t+ \\
+\frac{1}{L_{x} L_{y} L_{z}} \int_{0}^{L_{x}} \int_{0}^{L_{y} L_{z}} \int_{0} f_{\Phi V}(x, y, z) d z d y d x .
\end{aligned}
$$

The above substitution leads to obtaining equation for parameter $\alpha_{2 C}$. Solution of the equation will be different for different values of parameter $\gamma$. We used the second-order approximations framework method of averaging of function corrections with decreased quantity of iterative steps for analysis of spatio-temporal distributions of concentrations of dopant and radiation defects and temperature. The second-order approximation is usually a sufficient approximation to obtain qualitative and some quantitative results. We checked our analytical results by numerical approaches.

\section{Discussion}

Based on recently calculated relations we analyzed redistribution of dopant and radiation defects. Figures $2 a$ and $2 b$ show distributions of concentrations of infused (Fig. 2a) and implanted (Fig. $2 b$ ) dopants in channels and in nearest areas. We calculate the above distribution for larger value of dopant diffusion coefficient in doped materials in comparison with the analogous coefficient in undoped area. In this case it is possible to increase density of channels in transistors and, as a consequence, to decrease dimensions of multi-channel transistor. At the same time the considered approach of doping gives us possibility to increase homogeneity of concentrations of dopants in channels of the considered transistors. In this situation one can obtain increasing density of electrical current in the channels at fixed value of maximal heating of the doped material or to decrease length of channels. It should be noted, that using ion implantation gives us possibility to increase homogeneity of concentrations of dopants in channels and at the same time to decrease their quantities in nearest materials due to radiation-induced diffusion.

Analysis of changing of concentration of dopant in time shown necessity of optimization of annealing time. Reason of the optimization is too large diffusion depth of dopants from channels of transistors into nearest materials. Figs. 3 are illustrations of this situations. We used recently introduced criterion [9,10,18-24] to determine optimal value of annealing time. Framework the criterion we approximate real distribution of concentration of dopant by idealized step-wise function

$\psi(x, y, z)$. Framework the criterion we minimize the following mean-squared error to determine the optimal value of annealing time

$$
U=\frac{1}{L_{x} L_{y} L_{z}} \int_{0}^{L_{x}} \int_{0}^{L_{y}} \int_{0}^{L_{z}}[C(x, y, z, \Theta)-\psi(x, y, z)] d z d y d x .
$$




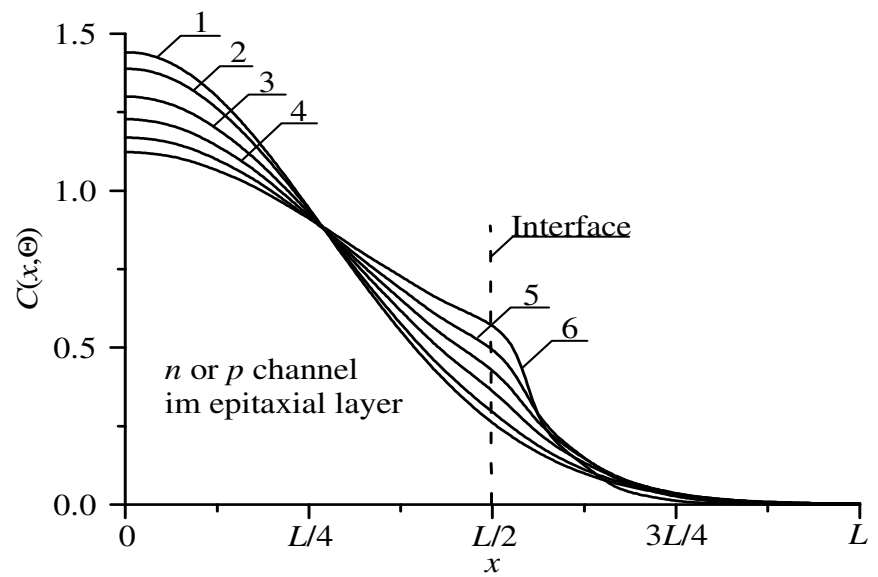

Fig. 2a. Spatial distributions of concentrations of infused dopant. Coordinate $x$ is perpendicular to interface between layers of heterostructure. Difference between values of dopant diffusion coefficient in layers of heterostructure increases with increasing of number of distributions.

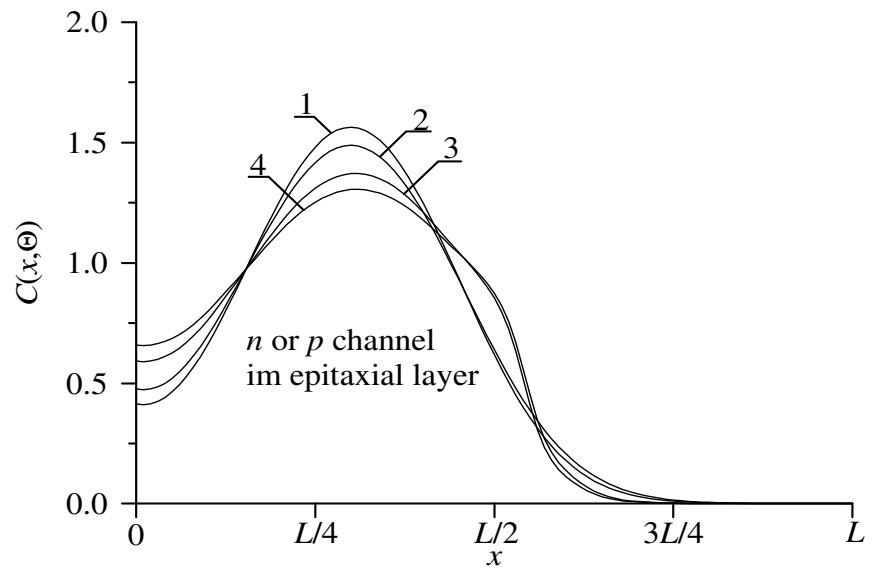

Fig. 2b. Spatial distributions of concentrations of infused dopant. Distributions 1 and 2 have been calculated in homogenous material for two values of annealing time $\left(\Theta=0,0048\left(L_{x}^{2}+L_{y}^{2}+L_{z}^{2}\right) / D_{0}\right.$ and $\Theta=0,0057$ $\left(L_{x}^{2}+L_{y}{ }^{2}+L_{z}^{2}\right) / D_{0}$, respectively). Distributions 3 and 4 have been calculated for heterostructure with two layers for the same annealing time

The considered distributions in Figs. 2 corresponds exactly to compromise annealing time.

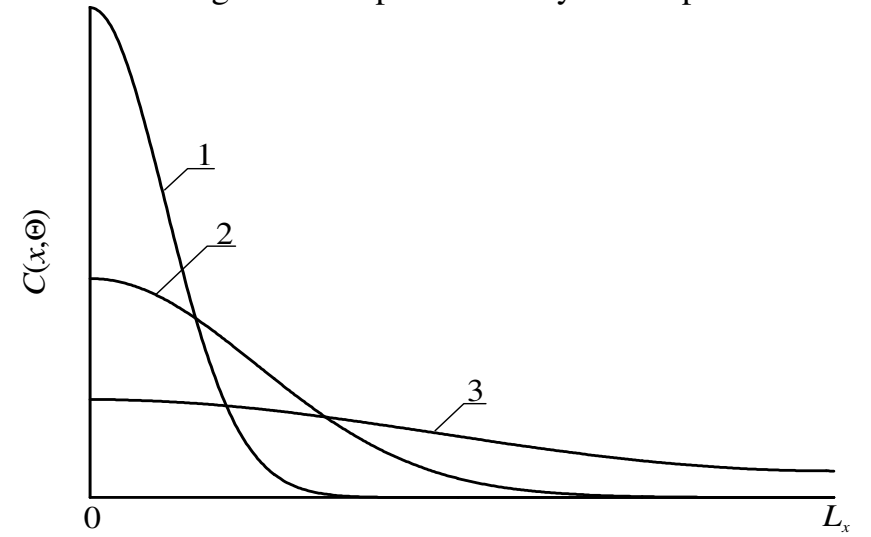

Fig.3a. Distributions of concentration of infused dopant on coordinate for different values of annealing time. Value of annealing time increases with increasing of number of curves 


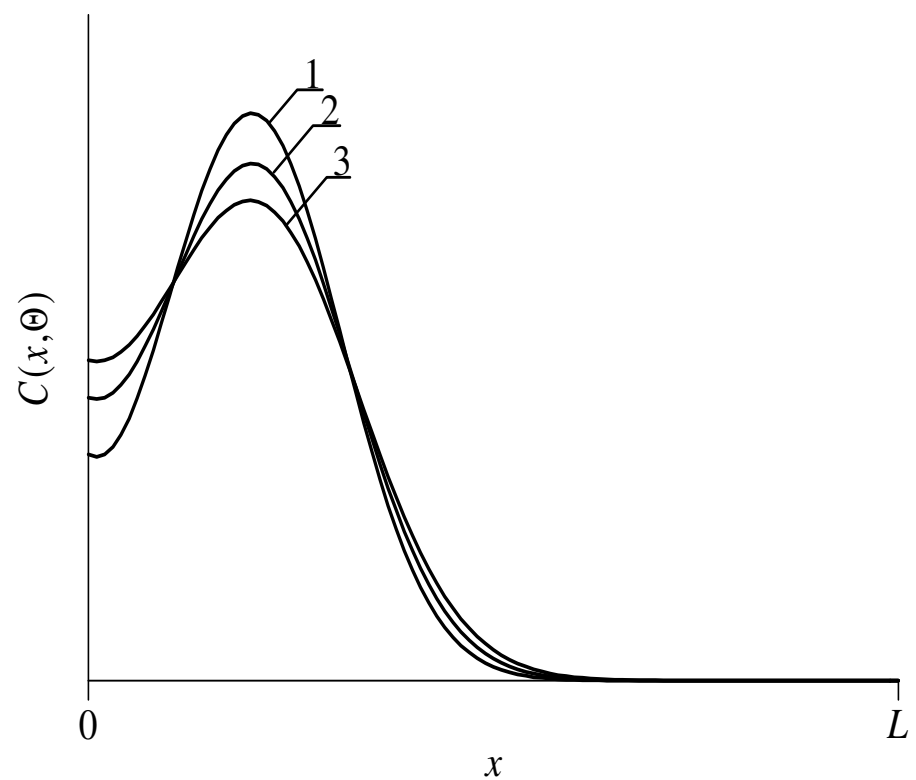

Fig. $3 a$. Distributions of concentration of implanted dopant on coordinate for different values of annealing time. Value of annealing time increases with increasing of number of curves

\section{ConClusions}

In this paper we introduce an approach for manufacturing a multi-channel heterotransistor. Several recommendations to optimize technological process for decreasing dimensions of transistor.

\section{ACKNOWLEDGEMENTS}

This work is supported by the agreement of August 27, 2013 № 02.B.49.21.0003 between The Ministry of education and science of the Russian Federation and Lobachevsky State University of Nizhni Novgorod and educational fellowship for scientific research of Government of Russian and of Nizhny Novgorod State University of Architecture and Civil Engineering.

\section{REFERENCES}

[1] N.I. Volokobinskaya, I.N. Komarov, T.V. Matioukhina, V.I. Rechetniko, A.A. Rush, I.V. Falina, A.S. Yastrebov. Investigation of technological processes of manufacturing of the bipolar power highvoltage transistors with a grid of inclusions in the collector region. Semiconductors. Vol. 35 (8). P. 974-978 (2001).

[2] G. Volovich. Modern chips UM3Ch class D manufactured by firm MPS. Modern Electronics. Issue 2. P. 10-17 (2006).

[3] M.V. Dunga, L. Chung-Hsun, X. Xuemei, D.D. Lu, A.M. Niknejad, H. Chenming. Modeling Advanced FET Technology in a Compact Model. IEEE Transactions on Electron Devices. Vol. 53 (9). P. 157-162 (2006).

[4] A. Kerentsev, V. Lanin. Constructive-technological features of MOSFET-transistors. Power Electronics. Issue 1. P. 34-38 (2008).

[5] O.A. Ageev, A.E. Belyaev, N.S. Boltovets, V.N. Ivanov, R.V. Konakova, Ya.Ya. Kudryk, P.M. Lytvyn, V.V. Milenin, A.V. Sachenko. Influence of displacement of the electron-hole equilibrium on the process of transition metals diffusion in GaAs. Semiconductors. Vol. 43 (7). P. 865-871 (2009).

[6] C. Senthilpari, K. Diwakar, A.K. Singh. Low Energy, Low Latency and High Speed Array Divider Circuit Using a Shannon Theorem Based Adder Cell Recent Patents on Nanotechnology. Vol. 3 (1). P. 61-72 (2009). 
[7] K.K. Ong, K.L. Pey, P.S. Lee, A.T.S. Wee, X.C. Wang, Y.F. Chong. Dopant distribution in the recrystallization transient at the maximum melt depth induced by laser annealing. Appl. Phys. Lett. 89 (17), 172111-172114 (2006).

[8] H.T. Wang, L.S. Tan, E. F. Chor. Pulsed laser annealing of Be-implanted GaN. J. Appl. Phys. Vol. 98 (9), 094901-094905 (2006).

[9] E.L. Pankratov. Redistribution of dopant during annealing of radiative defects in a multilayer structure by laser scans for production an implanted-junction rectifiers. Int. J. Nanoscience. Vol. 7 (4-5). P. 187-197 (2008).

[10] E.L. Pankratov. Decreasing of depth of implanted-junction rectifier in semiconductor heterostructure by optimized laser annealing. J. Comp. Theor. Nanoscience. Vol. 7 (1). P. 289-295 (2010).

[11] Yu.V. Bykov, A.G. Yeremeev, N.A. Zharova, I.V. Plotnikov, K.I. Rybakov, M.N. Drozdov, Yu.N. Drozdov, V.D. Skupov. Diffusion processes in semiconductor structures during microwave annealing. Radiophysics and Quantum Electronics. Vol. 43 (8-9). P. 749-755 (2003).

[12] H. Ryssel, I. Ruge. Ion implantation. B.G. Teubner, Stuttgart, 1978.

[13] V.L. Vinetskiy, G.A. Kholodar', Radiative physics of semiconductors. "Naukova Dumka", Kiev, 1979, in Russian.

[14] Z.Yu. Gotra. Technology of microelectronic devices (Radio and communication, Moscow, 1991).

[15] P.M. Fahey, P.B. Griffin, J.D. Plummer. Point defects and dopant diffusion in silicon. Rev. Mod. Phys. 1989. V. 61. № 2. P. 289-388.

[16] Yu.D. Sokolov. About the definition of dynamic forces in the mine lifting. Applied Mechanics. Vol.1 (1). P. 23-35 (1955).

[17] E.L. Pankratov. Dynamics of delta-dopant redistribution during heterostructure growth. The European Physical Journal B. Vol. 57 (3). P. 251-256 (2007).

[18] E.L. Pankratov. Influence of mechanical stress in a multilayer structure on spatial distribution of dopants in implanted-junction and diffusion-junction rectifiers. Mod. Phys. Lett. B. Vol. 24 (9). P. 867895 (2010).

[19] E.L. Pankratov. Dopant Diffusion Dynamics and Optimal Diffusion Time as Influenced by DiffusionCoefficient Nonuniformity Russian Microelectronics. 2007. V.36 (1). P. 33-39.

[20] E.L. Pankratov. Redistribution of dopant during annealing of radiative defects in a multilayer structure by laser scans for production an implanted-junction rectifiers. Int. J. Nanoscience. Vol. 7 (4-5). P. 187-197 (2008).

[21] E.L. Pankratov. Decreasing of depth of implanted-junction rectifier in semiconductor heterostructure by optimized laser annealing. J. Comp. Theor. Nanoscience. Vol. 7 (1). P. 289-295 (2010).

[22] E.L. Pankratov, E.A. Bulaeva. Application of native inhomogeneities to increase compactness of vertical field-effect transistors. J. Comp. Theor. Nanoscience. Vol. 10 (4). P. 888-893 (2013).

[23] E.L. Pankratov, E.A. Bulaeva. An approach to manufacture of bipolar transistors in thin film structures. On the method of optimization. Int. J. Micro-Nano Scale Transp. Vol. 4 (1). P. 17-31 (2014).

[24] E.L. Pankratov, E.A. Bulaeva. Increasing of sharpness of diffusion-junction heterorectifier by using radiation processing. Int. J. Nanoscience. Vol. 11 (5). P. 1250028-1250035 (2012).

\section{Authors:}

Pankratov Evgeny Leonidovich was born at 1977. From 1985 to 1995 he was educated in a secondary school in Nizhny Novgorod. From 1995 to 2004 he was educated in Nizhny Novgorod State University: from 1995 to 1999 it was bachelor course in Radiophysics, from 1999 to 2001 it was master course in Radiophysics with specialization in Statistical Radiophysics, from 2001 to 2004 it was $\mathrm{PhD}$ course in Radiophysics. From 2004 to 2008 E.L. Pankratov was a leading technologist in Institute for Physics of Microstructures. From 2008 to 2012 E.L. Pankratov was a senior lecture/Associate Professor of Nizhny Novgorod State University of Architecture and Civil Engineering. Now E.L. Pankratov is in his Full Doctor course in Radiophysical Department of Nizhny Novgorod State University. He has 110 published papers in area of his researches.

Bulaeva Elena Alexeevna was born at 1991. From 1997 to 2007 she was educated in secondary school of village Kochunovo of Nizhny Novgorod region. From 2007 to 2009 she was educated in boarding school "Center for gifted children". From 2009 she is a student of Nizhny Novgorod State University of Architecture and Civil Engineering (spatiality "Assessment and management of real estate"). At the same time she 
International Journal of Recent advances in Physics (IJRAP) Vol.4, No.4, November 2015

is a student of courses "Translator in the field of professional communication" and "Design (interior art)" in the University. E.A. Bulaeva was a contributor of grant of President of Russia (grant № MK548.2010.2). She has 74 published papers in area of her researches.

\section{APPENDIX}

$$
\begin{aligned}
& A_{a b i j}=\frac{1}{\Theta L_{x} L_{y} L_{z}} \int_{0}^{\Theta}(\Theta-t) \int_{0}^{L_{x}} \int_{0}^{L_{y} L_{z}} \int_{0} k_{a, b}(x, y, z, T) I_{1}^{i}(x, y, z, t) V_{1}^{j}(x, y, z, t) d z d y d x d t, \\
& B_{4}=A_{I V 00}^{2} A_{I V 00}^{2}-2\left(A_{I V 00}^{2}-A_{I I 00} A_{V V 00}\right)^{2}, B_{3}=A_{I V 00} A_{I V 00}^{2}+A_{I V 01} A_{I V 00}^{3}+A_{I V 00} A_{I I 10} A_{I V 00}^{2}- \\
& -4\left(A_{I V 00}^{2}-A_{I I 00} A_{V V 00}\right)\left[2 A_{I V 01} A_{I V 00}+2 A_{I V 00}\left(1+A_{I V 01}+A_{I I 10}\right)-2 A_{I I 00}\left(A_{I V 10}+A_{V V 10}+1\right)\right]- \\
& -4 A_{I V 10} A_{I V 10} A_{I I 00} A_{I V 00}^{2}+2 A_{I V 00} A_{I V 01} A_{I V 00}^{2}, B_{2}=A_{I V 00}^{2}\left\{\left(1+A_{I V 01}+A_{I I 10}\right)^{2}+A_{I V 00}^{2} A_{I V 01}^{2}-A_{I I 00} \times\right. \\
& \times 4\left[A_{I V 11}-A_{I I 20}-\frac{1}{L_{x} L_{y} L_{z}} \int_{0}^{L_{x}} \int_{0}^{L_{y} L_{z}} \int_{0} f_{I}(x, y, z) d z d y d x\right]+2 A_{I V 00} A_{I V 01}\left(A_{I V 00}+A_{I V 00} A_{I V 01}+\right. \\
& \left.\left.+A_{I V 00} A_{I I 10}-4 A_{I V 10} A_{I I 00}\right)\right\}\left[2 A_{I V 01} A_{I V 00}+2 A_{I V 00}\left(1+A_{I V 01}+A_{I I 10}\right)-2 A_{I I 00}\left(A_{I V 10}+A_{V V 10}+\right.\right. \\
& +1)]^{2}+2\left[A_{I V 01}\left(1+A_{I V 01}+A_{I I 10}\right)+\frac{2}{L_{x} L_{y} L_{z}} \int_{0}^{L_{x}} \int_{0}^{L_{y} L_{z}} \int_{0} f_{V}(x, y, z) d z d y d x-2\left(A_{V V 20}-A_{I V 11}\right) \times\right. \\
& \left.\times A_{I I 00}+A_{I V 01}\left(1+A_{I V 01}+A_{I I 10}\right)\right]\left[2 A_{I V 00}\left(1+A_{I V 01}+A_{I I 10}\right)-2 A_{I I 00}\left(A_{I V 10}+A_{V V 10}+1\right)+\right. \\
& \left.\left.+2 A_{I V 01} A_{I V 00}\right]\right\}, B_{1}=2 A_{I V 00} A_{I V 01}\left(1+A_{I V 01}+A_{I I 10}\right)^{2}-8 A_{I V 00} A_{I V 01} A_{I I 00}\left[A_{I V 11}-A_{I I 20}-\right. \\
& \left.-\frac{1}{L_{x} L_{y} L_{z}} \int_{0}^{L_{x}} \int_{0}^{L_{y} L_{z}} \int_{0} f_{I}(x, y, z) d z d y d x\right]+\left(A_{I V 00}+A_{I V 00} A_{I V 01}+A_{I V 00} A_{I I 10}-4 A_{I V 10} A_{I I 00}\right) \times \\
& \times A_{I V 01}^{2}-2\left[\frac{2 A_{I I 00}}{L_{x} L_{y} L_{z}} \int_{0}^{L_{x}} \int_{0}^{L_{y} L_{z}} \int_{0} f_{I}(x, y, z) d z d y d x+A_{I V 01}\left(1+A_{I V 01}+A_{I I 10}\right)-\left(A_{V V 20}-A_{I V 11}\right) \times\right.
\end{aligned}
$$


International Journal of Recent advances in Physics (IJRAP) Vol.4, No.4, November 2015

$$
\begin{aligned}
& \left.\times 2 A_{I I 00}+A_{I V 01}\left(1+A_{I V 01}+A_{I I 10}\right)\right]\left[2 A_{I V 00}\left(1+A_{I V 01}+A_{I I 10}\right)-2 A_{I I 00}\left(A_{I V 10}+A_{V V 10}+1\right)+\right. \\
& \left.+2 A_{I V 01} A_{I V 00}\right], B_{0}=4 A_{I I 00} A_{I V 01}^{2}\left[A_{I I 20}+\frac{1}{L_{x} L_{y} L_{z}} \int_{0}^{L_{x}} \int_{0}^{L_{y} L_{z}} \int_{0} f_{I}(x, y, z) d z d y d x-A_{I V 11}\right]- \\
& -\left[\frac{2 A_{I I 00}}{L_{x} L_{y} L_{z}} \int_{0}^{L_{L} L_{y} L_{z}} \int_{0} \int_{0} f_{V}(x, y, z) d z d y d x+A_{I V 01}\left(1+A_{I V 01}+A_{I I 0}\right)-2 A_{I I 00}\left(A_{V V 20}-A_{I V 11}\right)+\right. \\
& \left.+A_{I V 01}\left(1+A_{I V 01}+A_{I I 10}\right)\right]^{2}+A_{I V 01}^{2}\left(A_{I V 01}+A_{I I 10}+1\right)^{2}, y=\sqrt[3]{\sqrt{q^{2}+p^{3}}-q}+B_{2} / 6- \\
& -\sqrt[3]{\sqrt{q^{2}+p^{3}}+q}, q=\frac{1}{8}\left[\frac{B_{2}^{3}}{27}+B_{0}\left(4 B_{2}-B_{3}^{2}\right)-B_{1}^{2}+\frac{B_{2}}{6}\left(2 B_{1} B_{3}-8 B_{0}\right)\right], A=\sqrt{8 y+B_{3}^{2}-4 B_{2}}, \\
& p=\left[3\left(2 B_{1} B_{3}-8 B_{0}\right)-2 B_{2}^{2}\right] / 72 .
\end{aligned}
$$

